\title{
Magnetic quantum phase transition of cold atoms in an optical lattice
}

\author{
Peng-Bin He, ${ }^{1,2}$ Qing Sun, ${ }^{2}$ Peng Li, ${ }^{3}$ Shun-Qing Shen, ${ }^{3}$ and W. M. Liu ${ }^{2}$ \\ ${ }^{1}$ Micro-Nano Technologies Research Center, College of Physics and Microelectronics Science, Hunan University, \\ Changsha 410082, China \\ ${ }^{2}$ Beijing National Laboratory for Condensed Matter Physics, Institute of Physics, Chinese Academy of Sciences, Beijing 100080, China \\ ${ }^{3}$ Department of Physics, The University of Hong Kong, Pokfulam Road, Hong Kong, China
}

(Received 14 March 2007; published 16 October 2007)

\begin{abstract}
We propose a scheme to investigate the magnetic phase transition of cold atoms confined in an optical lattice. We also demonstrate how to get coupled two-leg spin ladders which display a phase transition from a spin liquid to magnetic ordered state in two-dimensional optical lattice. An experimental protocol is further designed for observing this phenomenon.
\end{abstract}

DOI: 10.1103/PhysRevA.76.043618

PACS number(s): 03.75.Ss, 03.75.Lm, 03.75.Hh

\section{INTRODUCTION}

Cold atoms in optical lattices provide a useful experimental means to investigate quantum many-body systems due to the advantage of cleanness and controllability. Extensive works have been done in the past few years, such as implementing quantum phase transitions from a superfluid to a Mott insulator [1,2], simulating high- $T_{c}$ superconductivity [3], and realizing various Hubbard models and spin models $[4,5]$. By adjusting the amplitudes and propagation directions of laser beams, a variety of geometries of optical lattice are generated $[6,7]$. This technique offers the possibility for constructing spin systems including spin chains [8-10], kagome lattices [11], and spin ladders $[8,12,13]$. In experiments, except for cold Bose atoms, Fermi atoms in optical lattices have also been realized recently, such as ${ }^{40} \mathrm{~K}$ in onedimensional (1D) [14] and 3D [15] lattices, ${ }^{6} \mathrm{Li}$ in 3D lattices [16], and a variety of interesting phenomena-for example, Bloch oscillations, band insulators, and superfluidity-were reported.

It was known that spin ladders may provide a transition from 1D chains to 2D lattices [17]. A class of cuprates can be described by antiferromagnetic spin ladders with spin $1 / 2$ [18-21]. Applying a magnetic field or pressure, these cuprates show a phase transition from a spin liquid state to several ordered magnetic states. Strongly correlated cold atoms in optical lattices provide a way to observe this quantum phase transition.

In this paper, we propose an optical lattice setup to produce 2D coupled spin ladders to illustrate a quantum phase transition, from spin-dimerized phase to magnetically ordered phase, and further discuss experimental conditions which can be realized by adjusting the lattice parameters. Compared to cuprates, optical lattices are clean and easily controllable. Manipulating the amplitudes and wave vectors of laser beams, the coupling between spins can be adjusted in a wide range.

\section{OPTICAL LATTICES AND SPIN LADDERS}

A 2D superlattice is formed by superimposing a standing wave along the $x$ direction with twice the period of a $2 \mathrm{D}$ optical lattice, which was generated by two perpendicular standing waves, as illustrated in Figs. 1(a) and 1(b). Adjusting the intensities of the three standing waves, the tunneling and potential barriers along the $x$ and $y$ direction can be well controlled. For sufficiently large intensities of the laser, the on-site interaction strength is much larger than the kinetic energy so that the lattice-atom system can be in the Mott insulating phase at a commensurate filling. We will consider an equal-mixing two-component fermionic system and assume that the lattice has been loaded with one atom per lattice site. This is possible by using the coherent filtering scheme proposed in Ref. [22].

In general, the Hamiltonian of interacting cold atom gas in an optical lattice is written as
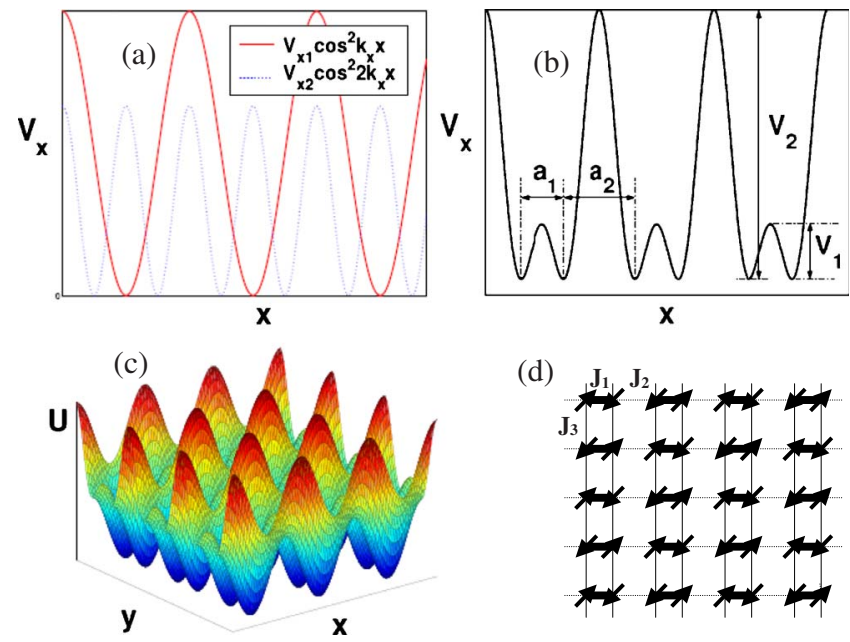

(d)

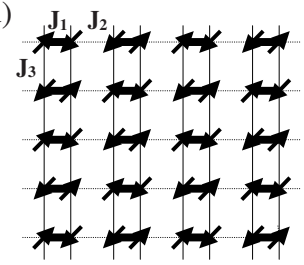

FIG. 1. (Color online) (a) Two selected optical potentials along the $x$ direction, where the potential $V_{x 2}$ (dotted line) has twice the period of $V_{x 1}$ (solid line). (b) Total potential in the $x$ axis, where $a_{1}$, $a_{2}, V_{1}$, and $V_{2}$ are the widths and heights of barriers of intradimers and interdimers, respectively. (c) Landscape of potential in the $x-y$ plane. (d) Geometry of the 2D coupled spin ladders, where $J_{1}, J_{2}$, and $J_{3}$ are intradimer, interdimer, and interladder spin-spin couplings, respectively. 


$$
\begin{aligned}
H= & \sum_{\sigma} \int d \mathbf{r} \hat{\Psi}_{\sigma}^{\dagger}(\mathbf{r})\left[-\frac{\hbar^{2}}{2 m} \nabla^{2}+U(\mathbf{r})\right] \hat{\Psi}_{\sigma}(\mathbf{r}) \\
& +\sum_{\sigma \sigma^{\prime}} \frac{g}{2} \int d \mathbf{r} \hat{\Psi}_{\sigma}^{\dagger}(\mathbf{r}) \hat{\Psi}_{\sigma^{\prime}}^{\dagger}(\mathbf{r}) \hat{\Psi}_{\sigma^{\prime}}(\mathbf{r}) \hat{\Psi}_{\sigma}(\mathbf{r}),
\end{aligned}
$$

where $g=4 \pi a_{s} \hbar / m$ and $a_{s}$ is the $s$-wave scattering length. The potential of optical lattice has the form

$$
\begin{aligned}
U(\mathbf{r})= & V_{x 1} \cos ^{2}\left(k_{x} x\right)+V_{x 2} \cos ^{2}\left(2 k_{x} x\right)+V_{y} \cos ^{2}\left(k_{y} y\right) \\
& +\left(m \omega_{z}^{2} z^{2}\right) / 2+V_{x 1}^{2} /\left(16 V_{x 2}\right)-V_{x 1} / 2,
\end{aligned}
$$

where $V_{x 1}$ and $V_{x 2}$ are the barrier heights of the two standing waves along the $x$ direction, $V_{y}$ is that along the $y$ direction, $k_{x}$ and $k_{y}$ are the two components of wave vector along the $x$ and $y$ directions, and $\omega_{z}$ is the harmonic frequency of the potential in the $z$ direction. The last two terms make the potential value zero in the bottom of every minitrap. To simulate a $2 \mathrm{D}$ coupled two-leg spin ladder, $4 V_{x 2}>V_{x 1}$ is necessary. The heights of the two potential barriers in the $x$ direction are $V_{1}=\left(4 V_{x 2}-V_{x 1}\right)^{2} /\left(16 V_{x 2}\right)$ and $V_{2}=\left(4 V_{x 2}\right.$ $\left.+V_{x 1}\right)^{2} /\left(16 V_{x 2}\right)$. The widths of the two barriers in the $x$ direction and the one in the $y$ direction are $a_{1}$ $=\operatorname{arcos}\left(V_{x 1} / 4 V_{x 2}\right) / k_{x}, \quad a_{2}=\left[\pi-\operatorname{arcos}\left(V_{x 1} / 4 V_{x 2}\right)\right] / k_{x}$, and $b$ $=\pi / k_{y}$. The geometry of the optical lattice is displayed in Fig. 1(b).

In the bottom of the trap, we take the harmonic approximation and the frequencies in the $x$ and $y$ directions are $\omega_{x}^{2}$ $=\left(16 V_{x 2}^{2}-V_{x 1}^{2}\right) k_{x}^{2} /\left(2 m V_{x 2}\right)$ and $\omega_{y}^{2}=2 V_{y} k_{y}^{2} / m$, respectively. When the on-site interaction and thermal fluctuation are much smaller than the excited energy of the second band, all atoms are located in the lowest band. The field operators can be expanded as $\hat{\Psi}_{\sigma}(\mathbf{r})=\Sigma_{i, j} c_{i, j, \sigma} w\left(\mathbf{r}-\mathbf{r}_{i, j}\right)$, and $w\left(\mathbf{r}-\mathbf{r}_{i, j}\right)$ is the ground-state function of the harmonic oscillators, $w(\mathbf{r})$ $=[m \bar{\omega} /(\pi \hbar)]^{3 / 4} e^{m\left(\omega_{x} x^{2}+\omega_{y} y^{2}+\omega_{z} z^{2}\right) /(2 \hbar)} \cdot c_{i, j, \sigma}$ are the annihilation operators for spin- $\sigma$ atoms localized at the site labeled by $(i, j)$, and $\bar{\omega}$ represents the geometric mean of frequencies, $\bar{\omega}=\left(\omega_{x} \omega_{y} \omega_{z}\right)^{1 / 3}$. Substituting the field operator into the Hamiltonian and integrating, we obtain an equivalent 2D Hubbard model

$$
\begin{aligned}
H= & -\sum_{i, j, \sigma}\left(t_{1} c_{2 i, j, \sigma}^{\dagger} c_{2 i+1, j, \sigma}+t_{2} c_{2 i+1, j, \sigma}^{\dagger} c_{2 i+2, j, \sigma}+\text { H.c. }\right) \\
& -\sum_{i, j, \sigma}\left(t_{3} c_{i, j, \sigma}^{\dagger} c_{i+1, j, \sigma}+\text { H.c. }\right)+U \sum_{i, j, \sigma} n_{i, j, \sigma} n_{i, j, \bar{\sigma}},
\end{aligned}
$$

where $\sigma= \pm 1 / 2$ represents the two states of cold atoms and $\bar{\sigma}=-\sigma$. The hopping and repulsive parameters are written as $t_{1}=e^{-m \omega_{x} a_{1}^{2} / 4 \hbar(}\left(t_{0}+\frac{m \omega_{x}^{2} a_{1}^{2}}{8}+\frac{V_{x 1}}{2} e^{-\hbar k_{x}^{2} / m \omega_{x}}\right), t_{2}=e^{-m \omega_{x} a_{2}^{2} / 4 \hbar(}\left(t_{0}+\frac{m \omega_{x}^{2} a_{2}^{2}}{8}\right.$ $-\frac{V_{x 1}}{2} e^{\left.-\hbar k_{x}^{2} / m \omega_{x}\right)}$, and $t_{3}=e^{-m \omega_{y} b^{2} / 4 \hbar\left(t_{0}+\frac{m \omega_{y}^{2} b^{2}}{8}\right.}-\frac{V_{x 1}}{2} e^{\left.-\hbar k_{x}^{2} / m \omega_{x}\right)}$, where $\quad t_{0}=-\frac{\hbar}{4}\left(\omega_{x}+\omega_{y}+2 \omega_{z}\right)-\frac{V_{x 2}}{2}\left(1+e^{-4 \hbar k_{x}^{2}} / m \omega_{x}\right)-\frac{V_{y}}{2}(1$ $+e^{\left.-\hbar k_{y}^{2} / m \omega_{y}\right)}-\frac{V_{x 1}^{2}}{16 V_{x 2}}$ and $U=\sqrt{\frac{2}{\pi}} \hbar \bar{\omega} a_{s} / \bar{a}_{0}$, with $\bar{a}_{0}=\sqrt{\hbar /(m \bar{\omega})}$.

In the regime of strong coupling, $U \gg t$, the half-filled Hubbard model is equivalent to a spin model approximately up to the order of $t_{1,2,3}^{2} / U$,

$$
\begin{aligned}
H= & \sum_{i, j}\left[J_{1} \mathbf{S}_{i, j}^{l} \cdot \mathbf{S}_{i, j}^{r}+J_{2} \mathbf{S}_{i, j}^{r} \cdot \mathbf{S}_{i+1, j}^{l}+J_{3}\left(\mathbf{S}_{i, j}^{l} \cdot \mathbf{S}_{i, j+1}^{l}\right.\right. \\
& \left.\left.+\mathbf{S}_{i, j}^{r} \cdot \mathbf{S}_{i, j+1}^{r}\right)\right],
\end{aligned}
$$

where $J_{m}=t_{m}^{2} /(4 U), m=1,2,3$, and $\mathbf{S}_{i, j}^{l, r}$ are the spin-1/2 operators on the left and right of the $(i, j)$ dimer. This Hamiltonian describes a 2D coupled spin ladder, as displayed in Fig. 1(d).

In real systems, an additional weak isotropic harmonic potential exists over the lattice [2]. This confinement brings an energy offset of each lattice site and leads to an effective local chemical potential. The superfluid-insulator phase diagram is influenced by this potential [1]. In the current case, the contribution of this potential only adds a term in Eq. (4):

$$
m \omega_{w h}^{2} \sum_{i, j, m}\left[\left(i-N_{1} / 2\right)^{2} a^{2}+\left(j-N_{2} / 2\right)^{2} b^{2}\right]\left(\mathbf{S}_{i, j}^{m}\right)_{z},
$$

where $m=l, r$ and $\omega_{w h}$ denotes the trapping frequency of the whole harmonic potential. $N_{1}$ and $N_{2}$ are the number of dimers along the $x$ and $y$ directions, respectively. Similarly, $a$ and $b$ represent the space of dimers. The effect of this whole harmonic potential corresponds to producing an effective magnetic field gradient in the $z$ direction. For a typical optical lattice, this potential is very weak; for example, in Ref. [2], the trapping frequency is about $65 \mathrm{~Hz}$. For a lattice with $\sim 100$ sites in a single direction, the Zeeman energy for atoms in different sites varies from $\sim 10^{-5} E_{r}$ to $\sim 10^{-2} E_{r}$. The magnitude is much smaller than the coupling energy. Thus, this additional harmonic potential is ignored in the following discussion.

\section{PHASE TRANSITION}

In the following, we consider repulsive spin-1/2 atoms $[23,24]$ and focus on the magnetic properties of the system described in Eq. (4). By adjusting the optical lattice parameters, we can reach the regime that $J_{1}>J_{2}\left(J_{3}\right)$. In this case, the bond operator method [25] is appropriate to study the properties of the system at low temperature. It describes well the dimerized phase and several magnetically ordered phases of dimer-based antiferromagnets. For a dimer of two 1/2 spins, the Hilbert space can be spanned by four states: one spin singlet $|s\rangle=(|\uparrow \downarrow\rangle-|\downarrow \uparrow\rangle) / \sqrt{2}$ and three spin triplets $\left|t_{x}\right\rangle=-(|\uparrow \uparrow\rangle-|\downarrow \downarrow\rangle) / \sqrt{2}, \quad\left|t_{y}\right\rangle=i(|\uparrow \uparrow\rangle+|\downarrow \downarrow\rangle) / \sqrt{2}$ and $\left|t_{z}\right\rangle$ $=(|\uparrow \downarrow\rangle+|\downarrow \uparrow\rangle) / \sqrt{2}$. Bond operators $s^{\dagger}$ and $t_{\alpha}^{\dagger}(\alpha=x, y, z)$ are introduced to generate the singlet and triplet states out of the vacuum $|0\rangle,|s\rangle=s^{\dagger}|0\rangle,\left|t_{x}\right\rangle=t_{x}^{\dagger}|0\rangle,\left|t_{y}\right\rangle=t_{y}^{\dagger}|0\rangle$, and $\left|t_{z}\right\rangle=t_{z}^{\dagger}|0\rangle$. The bond operators $s$ and $t_{\alpha}$ are assumed to satisfy the bosonic communication relations with a constraint of single occupancy at each bond, $s_{i, j}^{\dagger} s_{i, j}+t_{i, j}^{\alpha \dagger} t_{i, j}^{\alpha} \equiv 1$.

In terms of these four operators, the two spin operators in a dimer can be expressed as $\left(S_{i, j}^{l}\right)_{\alpha}=\left(s_{i, j}^{\dagger} t_{i, j}^{\alpha}+t_{i, j}^{\alpha \dagger} s_{i, j}\right.$ $\left.-i \epsilon_{\alpha \beta \gamma} t_{i, j}^{\beta \dagger} t_{i, j}^{\gamma}\right) / 2$ and $\left(S_{i, j}^{r}\right)_{\alpha}=\left(-s_{i, j}^{\dagger} t_{i, j}^{\alpha}-t_{i, j}^{\alpha \dagger} s_{i, j}-i \epsilon_{\alpha \beta \gamma} t_{i, j}^{\beta \dagger} t_{i, j}^{\gamma}\right) / 2$, respectively, where $\alpha, \beta$, and $\gamma$ represent $x, y$, and $z$, and $\epsilon$ is the fully antisymmetric Kronecker symbol. Repeated indices are summed over. Under the above transformation, the spin Hamiltonian, Eq. (4) can be transferred into a bosonic one. The constraint condition can be realized by introducing the 


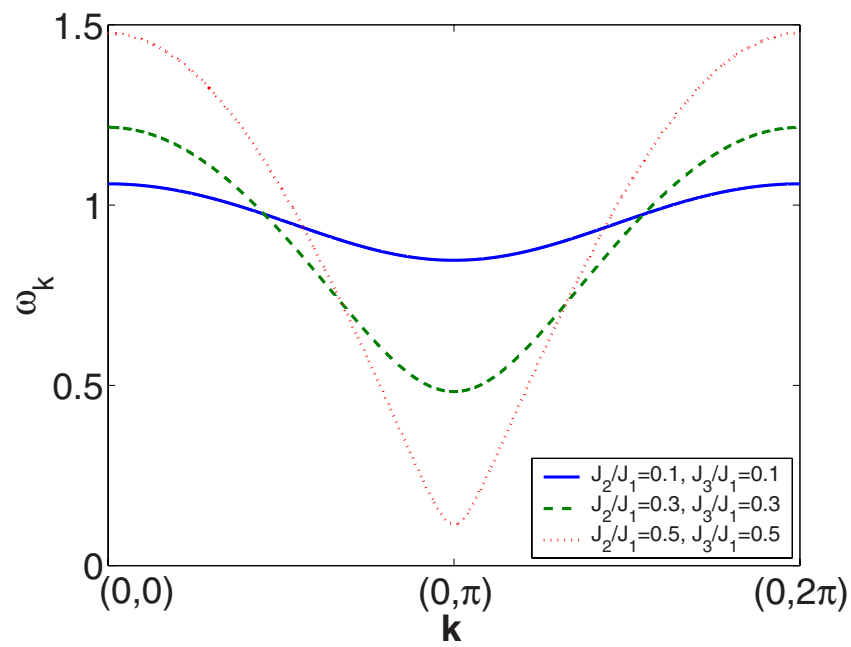

FIG. 2. (Color online) The energy spectrums of different interdimer couplings in the spin liquid phase. The solid, dashed, and dotted lines correspond to coupling strength $J_{2} / J_{1}=J_{3} / J_{1}=0.1,0.3$, 0.5 , respectively. The optical lattice parameters $V_{x 1}=5 E_{r}, V_{x 2}$ $=20 E_{r}$, and $V_{y}=28 E_{r}$ for $J_{2} / J_{1}=J_{3} / J_{1}=0.1 ; V_{x 1}=16 E_{r}, V_{x 2}=10 E_{r}$, and $V_{y}=6 E_{r}$ for $J_{2} / J_{1}=J_{3} / J_{1}=0.3 ; V_{x 1}=20 E_{r}, V_{x 2}=12 E_{r}$, and $V_{y}$ $=7 E_{r}$ for $J_{2} / J_{1}=J_{3} / J_{1}=0.5 . E_{r}=\frac{\hbar^{2} k_{x}^{2}}{2 m}$, where $m$ is the mass of the ${ }^{6} \mathrm{Li}$ atom, $a_{s}=2.41 \mathrm{~nm}, m=9.99 \times 10^{-26} \mathrm{~kg}, k_{x}=2 k_{y}=5.93 \mu \mathrm{m}^{-1}$, and $\omega_{z}=6600 \mathrm{~Hz} . \omega_{k}$ is measured in units of $J_{1} . k_{x}$ and $k_{y}$ are measured in units of $1 / a$ and $1 / b$, respectively. In the following figures, the atom parameters and laser wavelengths are the same.

Lagrange multipliers $\mu_{i, j}$ in the Hamiltonian, $-\sum \mu_{i, j}\left(s_{i, j}^{\dagger} s_{i, j}\right.$ $i, j$ $\left.+t_{i, j}^{\alpha \dagger} t_{i, j}^{\alpha}-1\right)$. Performing the Fourier transformation $t_{i, j}^{\alpha}$ $=1 / \sqrt{N} \Sigma_{\mathbf{k}} t_{\mathbf{k}}^{\alpha} e^{i \mathbf{k} \cdot \mathbf{r}_{i, j},} s_{i, j}=1 / \sqrt{N} \Sigma_{\mathbf{k}} s_{\mathbf{k}} e^{i \mathbf{k} \cdot \mathbf{r}_{i, j}}$ ( $N$ is the number of dimers), and taking $s_{\mathbf{k}}=s_{\mathbf{k}}^{\dagger}=\widetilde{s}$ as that most of the $s$ are condensed in the condition $J_{1} \gg J_{2,3}$, we get $H=\Sigma_{\mathbf{k}}\left[\Gamma_{\mathbf{k}} t_{\mathbf{k}}^{\alpha \dagger} t_{\mathbf{k}}^{\alpha}\right.$ $\left.+\Delta_{\mathbf{k}}\left(t_{\mathbf{k}}^{\alpha \dagger} t_{-\mathbf{k}}^{\alpha \dagger}+t_{\mathbf{k}}^{\alpha} t_{-\mathbf{k}}^{\alpha}\right)\right]+H_{0}$, where $H_{0}=N\left(-3 J_{1} \tilde{s}^{2} / 4-\mu \tilde{s}^{2}+\mu\right)$, $\Gamma_{\mathbf{k}}=J_{1} / 4-\mu-\left(J_{2} / 2\right) \tilde{s}^{2} \cos \left(k_{x} a\right)+J_{3} \tilde{s}^{2} \cos \left(k_{y} b\right)$, and $\Delta_{\mathbf{k}}=$ $-\left(J_{2} / 4\right) \tilde{s}^{2} \cos \left(k_{x} a\right)+\left(J_{3} / 2\right) \tilde{s}^{2} \cos \left(k_{y} b\right)$, with $a=a_{1}+a_{2}$.

By using the Bogliubov transformation, the Hamiltonian can be diagonalized,

$$
H=\sum_{\mathbf{k}} \omega_{\mathbf{k}} \gamma_{\mathbf{k}}^{\alpha \dagger} \gamma_{\mathbf{k}}^{\alpha}+E
$$

The zero-temperature free energy is $E=N\left(-\frac{3 J_{1}}{4} \widetilde{s}^{2}-\mu \widetilde{s}^{2}+\mu\right)$ $+\frac{N}{(2 \pi)^{2}} \iint_{-\pi}^{\pi} d k_{x} d k_{y} \frac{3}{2}\left(\Omega_{k}-\Lambda_{k}\right), \quad$ where $\quad \Omega_{k}=\left(J_{1} / 4\right.$ $-\mu) \sqrt{1-m \cos k_{x}+n \cos k_{y}}, \quad \Lambda_{k}=\left(J_{1} / 4-\mu\right)\left[1-(m / 2) \cos k_{x}\right.$ $\left.+(n / 2) \cos k_{y}\right]$, with $m=J_{2} \tilde{s}^{2} /\left(J_{1} / 4-\mu\right)$ and $n=2 J_{3} \tilde{s}^{2} /\left(J_{1} / 4\right.$ $-\mu)$. The energy spectrum can be expressed as

$$
\omega_{k}=\left(J_{1} / 4-\mu\right) \sqrt{1-m \cos k_{x} a+n \cos k_{y} b} .
$$

Minimizing the free energy with respect to $\tilde{s}$ and $\mu$ gives $\partial E / \partial \widetilde{s}=0$ and $\partial E / \partial \mu=0$, which determines the parameters $\widetilde{s}$ and $\mu$ self-consistently. The energy gap between the ground state and the lowest point of spectrum is

$$
\Delta=\left(J_{1} / 4-\mu\right) \sqrt{1-(m+n)} .
$$

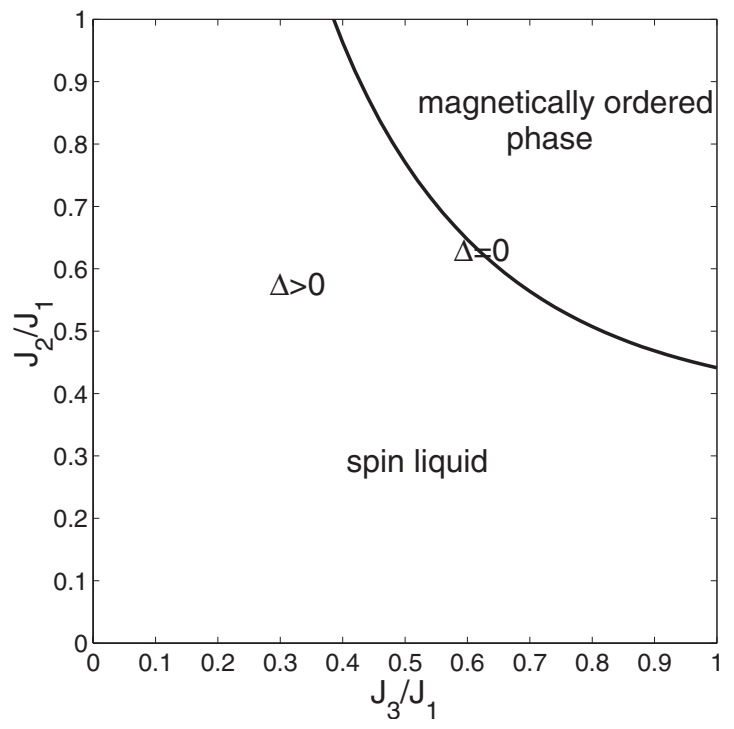

FIG. 3. Phase diagram controlled by the spin coupling strength. In the regime of the energy gap, $\Delta>0$, the system is spin disordered or in a spin liquid phase, while a magnetically ordered phase appears at $\Delta=0$. The phase boundary can be determined by the equation $\left(J_{2}+2 J_{3}\right) \tilde{s}^{2}=J_{1} / 4-\mu$, where the amplitude of condensate $\tilde{s}$ and chemical potential $\mu$ can be given by the variational method. $J_{1}, J_{2}$, and $J_{3}$ are functions of the lattice parameters, so the phase diagram is described by the amplitudes of standing waves in Fig. 4.

In the regime that $\Delta>0$, most spins form spin singlets in pairs. In this regime the system is spin disordered or named as spin liquid [26]. The three triplet modes are degenerate and massive in the spin liquid phase, as displayed in Fig. 2. When the interdimer coupling increases, the gap between excited and ground states decreases and the spectrum is more dispersive. At a critical point, the energy gap vanishes at the point $\mathbf{k}_{0}=(0, \pi / b)$ in the wave vector space. The $t_{\alpha}$ bosons condense and a magnetically ordered phase arises. The phase diagram of the spin liquid and magnetically ordered states is presented in Figs. 3 and 4. In Fig. 3, the controlling parameters are the ratio between the intradimer coupling and interdimer one. Due to the limitation of the bond operator method, the mean-field phase diagram needs to be improved when $J_{2} / J_{1}$ approaches 1 and $J_{3} / J_{1}$ approaches 0 . In Fig. 4, we gave the phase diagram in terms of lattice parameters. Adjusting the intensity of lasers, it is possible to realize the transition between two phases in optical lattices.

In the magnetically disordered phase, the excitation spectra of triplet bosons are threefold degenerate. Considering spontaneous symmetry breaking, we assume that $t_{z}$ bosons condensate as the energy gap $\Delta$ approaches zero. The ground state of a rung in the ordered phase can be expressed as $\left|\widetilde{\phi}_{0}\right\rangle=\frac{1}{\sqrt{1+\lambda^{2}}} \Pi_{i, j}\left(s_{i, j}^{\dagger}+\lambda e^{i \mathbf{k}_{0} \cdot \mathbf{R}_{i, j} z_{i, j}^{z \dagger}}\right)|0\rangle$, where $\lambda$ is the condensation amplitude of triplet bosons and $\mathbf{k}_{0}$ represents the inplane ordering wave vector (the gap begins to vanish in this point). The expected values of spin in this ground state are $\left\langle\left(S_{i, j}^{l}\right)_{x}\right\rangle=\left\langle\left(S_{i, j}^{r}\right)_{x}\right\rangle=\left\langle\left(S_{i, j}^{l}\right)_{y}\right\rangle=\left\langle\left(S_{i, j}^{r}\right)_{y}\right\rangle=0$ and $\left\langle\left(S_{i, j}^{l}\right)_{z}\right\rangle=-\left\langle\left(S_{i, j}^{r}\right)_{z}\right\rangle$ $=\left[\lambda /\left(1+\lambda^{2}\right)\right] \cos \left(\mathbf{k}_{0} \cdot \mathbf{R}_{i, j}\right)$. It is easy to see that all the dimers have a staggered magnetic structure and the magnitude of the spin expectation is related to the condensation of $t_{\alpha}$ bosons. 

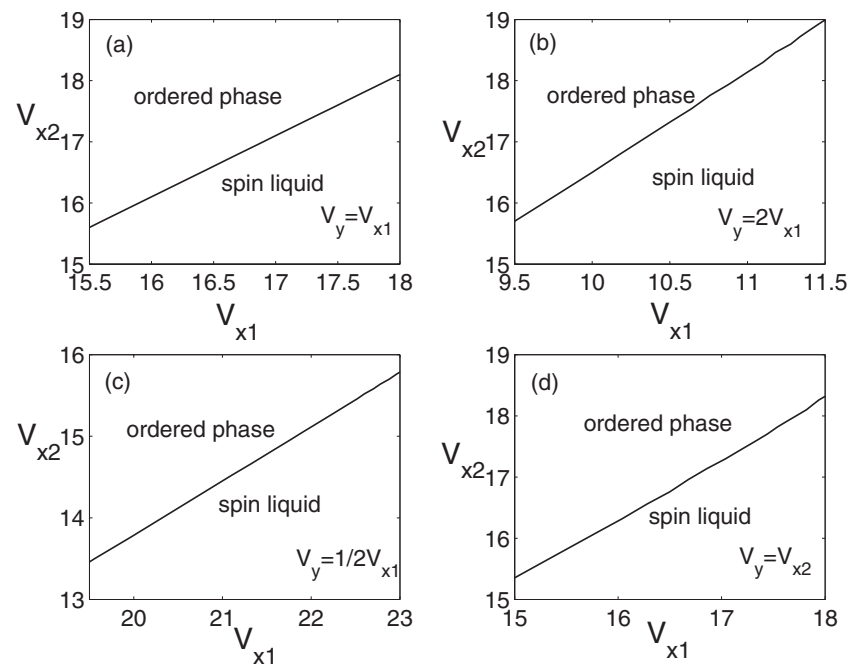

FIG. 4. Phase diagram in the $V_{x 1}-V_{x 2}$ plane for different $V_{y}$. The wave vector satisfies $k_{x}=2 k_{y} . V_{x 1}, V_{x 2}$, and $V_{y}$ are rescaled by the recoil energy $E_{r}$.

In order to describe the excitations of the ordered phase, we take the transformation $s_{i, j}^{\prime}=\left(s_{i, j}+\lambda e^{i \mathbf{k}_{0} \cdot \mathbf{R}_{i, j} t_{i, j}^{z}}\right)$ $/ \sqrt{1+\lambda^{2}}, \quad t_{i, j}^{\prime}{ }^{z}=\left(-\lambda e^{i \mathbf{k}_{0} \cdot \mathbf{R}_{i, j}} s_{i, j}+t_{i, j}^{z}\right) / \sqrt{1+\lambda^{2}}$, and $t_{i, j}^{\prime}{ }^{x}=t_{i, j}^{x}, t_{i, j}^{\prime}{ }^{y}$ $=t_{i, j}^{y}$. By performing this transformation and taking the Holstein-Primakoff expansion, $s_{i, j}^{\prime}=s_{i, j}^{\prime \dagger}=\left(1-1 / N t_{i, j}^{\prime}{ }^{\alpha^{\dagger}} t_{i, j}^{\prime}{ }^{\alpha}\right)^{1 / 2}$ [21], the Hamiltonian includes zero-, linear-, and quadraticorder terms in the $t^{\prime}$ bosonic operators. The disappearance of the linear term gives the value of $\lambda=\left(J_{2}+2 J_{3}-J_{1}\right) /\left(J_{2}+2 J_{3}\right.$ $+J_{1}$ ), which also minimizes the zero-order term. Finally, the Hamiltonian can be diagonalized as

$$
H=\sum_{\mathbf{k} \alpha} \omega_{\mathbf{k}}^{\alpha} \gamma_{\mathbf{k}}^{\alpha \dagger} \gamma_{\mathbf{k}}^{\alpha}+\sum_{\mathbf{k}}\left(\omega_{\mathbf{k}}^{x}+\frac{1}{2} \omega_{\mathbf{k}}^{z}-A_{\mathbf{k}}-\frac{1}{2} B_{\mathbf{k}}\right)+E_{0},
$$

where $\omega_{\mathbf{k}}^{x}=\omega_{\mathbf{k}}^{y}=\sqrt{A_{\mathbf{k}}^{2}-4 C_{\mathbf{k}}^{2}}, \quad \omega_{\mathbf{k}}^{z}=\sqrt{B_{\mathbf{k}}^{2}-4 D_{\mathbf{k}}^{2}}, \quad E_{0}=N\left(J_{1} / 4\right)\left(\lambda^{2}\right.$ $-3) /\left(1+\lambda^{2}\right)-\lambda^{2}\left(J_{2}+2 J_{3}\right) /\left(1+\lambda^{2}\right)^{2}, \quad$ and $\quad A_{\mathbf{k}}=\frac{J_{1}}{1+\lambda^{2}}+\left(J_{2}\right.$ $\left.+2 J_{3}\right) \frac{2 \lambda^{2}}{\left(1+\lambda^{2}\right)^{2}}+\frac{1-\lambda^{2}}{1+\lambda^{2}} M, \quad B_{\mathbf{k}}=J_{1} \frac{1-\lambda^{2}}{1+\lambda^{2}}+\left(J_{2}+2 J_{3}\right) \frac{4 \lambda^{2}}{\left(1+\lambda^{2}\right)^{2}}+\frac{\left(1-\lambda^{2}\right)^{2}}{\left(1+\lambda^{2}\right)^{2}} M$, and $\quad C_{\mathbf{k}}=\frac{M}{2}, \quad D_{\mathbf{k}}=\frac{\left(1-\lambda^{2}\right)^{2}}{\left(1+\lambda^{2}\right)^{2}} \frac{M}{2}, \quad$ with $\quad M=-\left(J_{2} / 2\right) \cos \left(k_{x} a\right)$ $+J_{3} \cos \left(k_{y} b\right)$. As a result, the energy spectrum in the magnetically ordered phase is obtained, as displayed in Fig. 5. In this phase, there are two degenerate spin-wave excitations restoring the breaking of rotational symmetry and one longitudinal mode which is gapped and corresponds to fluctuations in the amplitude of the moment.

\section{DETECTION OF THE PHASE TRANSITION}

In the spin liquid and magnetically ordered phases, there are gapped excitations which are triplet magnons in spin liquid and longitudinal modes in the ordered phase. These gaps can be probed by magnetic resonance [8]. When $\mu_{B} B \ll J_{1}$ $\left(\mu_{B}\right.$ is the magnetic moment of the alkali-metal atom and $B$ is the intensity of the oscillating magnetic field), the frequency of oscillating magnetic field has a resonant peak at $\omega=E_{\text {gap }} / \hbar$.

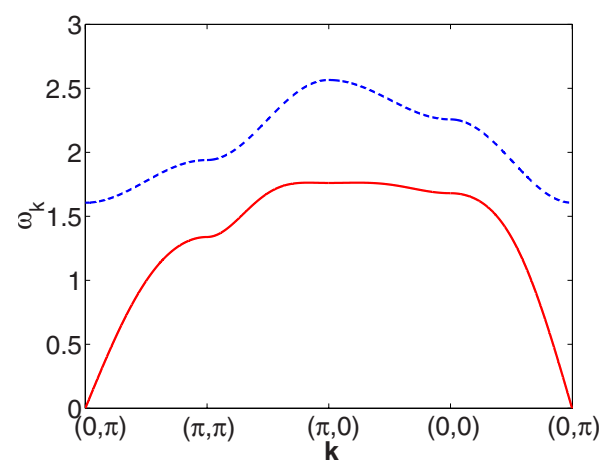

FIG. 5. (Color online) Energy spectrum in the magnetically ordered phase. The upper dashed line is the longitudinal mode, and the lower solid one is the doubly degenerate transverse mode. The coupling strength $J_{2} / J_{1}=J_{3} / J_{1}=0.7$. $\lambda=0.6$. The corresponding lattice parameters are $V_{x 1}=27 E_{r}, V_{x 2}=16 E_{r}$, and $V_{y}=10 E_{r}$. $\omega_{k}$ is measured in unit of $J_{1} . k_{x}$ and $k_{y}$ are measured in units of $1 / a$ and $1 / b$, respectively.

The excited spectrum is also a sign to distinguish the two phases. Two-photon Bragg scattering provides an effective method to measure the spectrum $[3,27]$. In such experiments two laser beams with different wave vectors and frequencies illuminate the atom cloud. The frequency difference is much smaller than the detuning of the two lasers from atomic resonance. The atoms absorb a photon from one beam and emit another photon into the other. By changing the angle between the two laser beams, we can tune the momentum transfer (momentum difference between the two beams). If the momentum and frequency differences match those of the dispersion relation of the spectrum, a resonant absorption of the probe light happens. Other methods for measuring spin correlation functions were proposed to distinguish the different magnetic phases [8].

\section{CONCLUSION}

In summary, 2D coupled spin ladders can be simulated by an optical superlattice. A phase transition from the spin liquid to magnetically ordered phase is possibly realized through adjusting the lattice parameters controlled by laser beams. Our results show that strongly correlated cold atoms in optical lattices provide a route to observe this quantum phase transition. Manipulating the amplitudes and wave vectors of laser beams, the coupling between spins can be adjusted in a wide range. Recent developments of controlling the cold atoms in optical lattices allow for the experimental investigation of our prediction in the future.

\section{ACKNOWLEDGMENTS}

We are grateful to S. Chen for helpful discussions. This work was supported by NSF of China under Grants Nos. 90403034, 90406017, 60525417, and 50602015, the NKBRSF of China under Grants Nos. 2005CB724508 and 2006CB921400, the 985 project of HNU, and the RGC of Hong Kong under Grant No.: HKU 7038/04P (SQS). 
[1] D. Jaksch, C. Bruder, J. I. Cirac, C. W. Gardiner, and P. Zoller, Phys. Rev. Lett. 81, 3108 (1998).

[2] M. Greiner et al., Nature (London) 415, 39 (2002).

[3] W. Hofstetter, J. I. Cirac, P. Zoller, E. Demler, and M. D. Lukin, Phys. Rev. Lett. 89, 220407 (2002).

[4] C. Honerkamp and W. Hofstetter, Phys. Rev. Lett. 92, 170403 (2004).

[5] D. Jakscha and P. Zoller, Ann. Phys. (N.Y.) 315, 52 (2005).

[6] P. B. Blakie et al., J. Phys. B 37, 1391 (2004).

[7] K. I. Petsas, A. B. Coates, and G. Grynberg, Phys. Rev. A 50, 5173 (1994).

[8] J. J. García-Ripoll, M. A. Martin-Delgado, and J. I. Cirac, Phys. Rev. Lett. 93, 250405 (2004).

[9] W. P. Zhang, Pu Han, C. Search, and P. Meystre, Phys. Rev. Lett. 88, 060401 (2002); Z. W. Xie, W. Zhang, S. T. Chui, and W. M. Liu, Phys. Rev. A 69, 053609 (2004).

[10] L. M. Duan, E. Demler, and M. D. Lukin, Phys. Rev. Lett. 91, 090402 (2003).

[11] L. Santos, M. A. Baranov, J. I. Cirac, H. U. Everts, H. Fehrmann, and M. Lewenstein, Phys. Rev. Lett. 93, 030601 (2004).

[12] P. Buonsante, V. Penna, and A. Vezzani, Phys. Rev. A 70, 061603(R) (2004); 72, 031602 (2005).

[13] S. Trebst, U. Schollwock, M. Troyer, and P. Zoller, Phys. Rev. Lett. 96, 250402 (2006).

[14] G. Modugno, F. Ferlaino, R. Hiedemann, G. Roati, and M. Inguscio, Phys. Rev. A 68, 011601(R) (2003); G. Roati, E. de Mirandes, F. Ferlaino, H. Ott, G. Modugno, and M. Inguscio, Phys. Rev. Lett. 92, 230402 (2004); L. Pezze, L. Pitaevskii, A. Smerzi, S. Stringari, G. Modugno, E. De Mirandes, F. Fer- laino, H. Ott, G. Roati, and M. Inguscio, ibid. 93, 120401 (2004).

[15] M. Köhl, H. Moritz, T. Stoferle, K. Günter, and T. Esslinger, Phys. Rev. Lett. 94, 080403 (2005); T. Stoferle, H. Moritz, K. Günter, M. Köhl, and T. Esslinger, ibid. 96, 030401 (2006).

[16] J. K. Chin et al., Nature (London) 443, 961 (2006).

[17] E. Dagotto and T. M. Rice, Science 271, 618 (1996).

[18] Sudha Gopalan, T. M. Rice, and M. Sigrist, Phys. Rev. B 49, 8901 (1994).

[19] B. Normand and T. M. Rice, Phys. Rev. B 54, 7180 (1996); 56, 8760 (1997).

[20] M. Vojta and K W. Becker, Phys. Rev. B 60, 15201 (1999); T. Sommmer et al., Eur. Phys. J. B 23, 329 (2001); M. Vojta and T. Ulbricht, Phys. Rev. Lett. 93, 127002 (2004).

[21] M. Matsumoto, B. Normand, T. M. Rice, and M. Sigrist, Phys. Rev. Lett. 89, 077203 (2002); Phys. Rev. B 69, 054423 (2004).

[22] P. Rabl, A. J. Daley, P. O. Fedichev, J. I. Cirac, and P. Zoller, Phys. Rev. Lett. 91, 110403 (2003).

[23] M. Rigol, A. Miramatsu, G. G. Batrouni, and R. T. Scalettar, Phys. Rev. Lett. 91, 130403 (2003).

[24] C. H. Lee, Phys. Rev. Lett. 93, 120406 (2004).

[25] S. Sachdev and R N. Bhatt, Phys. Rev. B 41, 9323 (1990).

[26] P. Li and S. Q. Shen, New J. Phys. 6, 160 (2004).

[27] J. Stenger, S. Inouye, A. P. Chikkatur, D. M. Stamper-Kurn, D. E. Pritchard, and W. Ketterle, Phys. Rev. Lett. 82, 4569 (1999); A. Imambekov, M. Lukin, and E. Demler, Phys. Rev. A 68, 063602 (2003). 